\title{
Surgical management of biliary cystadenoma and cystadenocarcinoma of the liver
}

\author{
Y.W. Chen, C.H. Li, Z. Liu, J.H. Dong, W.Z. Zhang and K. Jiang \\ Department of Hepatobiliary Surgery, Hepatobiliary Surgical Institute, \\ Chinese PLA General Hospital, Beijing, China \\ Corresponding author: K. Jiang \\ E-mail: JiangK301@126.com
}

Genet. Mol. Res. 13 (3): 6383-6390 (2014)

Received January 25, 2013

Accepted August 1, 2013

Published August 25, 2014

DOI http://dx.doi.org/10.4238/2014.August.25.1

\begin{abstract}
Biliary cystadenoma (BCA) and biliary cystadenocarcinoma (BCAC) are rare biliary duct neoplasms. This study investigated reasonable management strategies of cystic neoplasms in the liver. Charts of $39 \mathrm{BCA} / \mathrm{BCAC}$ patients ( 9 males, 30 female; median age $53.74 \pm 14.50$ years) who underwent surgery from January 1999 to December 2009 were reviewed retrospectively. Cyst fluid samples of $32 \mathrm{BCA} / \mathrm{BCAC}$ patients and 40 simple hepatic cyst patients were examined for the tumor markers carbohydrate associated antigen 19-9 (CA19-9) and carcinoembryonic antigen (CEA). The most frequent symptoms were abdominal pain $(\mathrm{N}=10)$, abdominal mass $(\mathrm{N}=7)$, abdominal distension $(\mathrm{N}=4)$, jaundice $(\mathrm{N}=2)$, and fever $(\mathrm{N}=3)$; the remaining patients showed no clinical symptoms. Liver resection $(\mathrm{N}$ $=17)$ or enucleation $(\mathrm{N}=22)$ was performed in the 39 patients. Ultimately, 35 patients were diagnosed with intrahepatic BCA and four patients were diagnosed with BCAC. The median CA19-9 level was significantly higher in $\mathrm{BCA} / \mathrm{BCAC}$ patients than in simple hepatic cyst patients. The median CEA levels in BCA/BCAC patients and controls were $6.83 \pm 2.43$ and $4.21 \pm 2.91 \mathrm{mg} / \mathrm{L}$, respectively. All symptoms were resolved after surgery, and only one BCAC patient showed recurrence. The incidence of intrahepatic cystic lesions was $1.7 \%$. Increased
\end{abstract}


CA19-9 levels in the cyst fluid is a helpful marker for distinguishing $\mathrm{BCA} / \mathrm{BCAC}$ from common simple cysts. The presence of coarse calcifications is suggestive of BCAC. Complete surgical removal of these lesions yielded satisfying long-term outcomes with a very low recurrence rate.

Key words: Biliary cystadenoma; Cystadenocarcinoma; Enucleation; Hepatic resection; CA19-9

\section{INTRODUCTION}

Intrahepatic biliary cystadenoma (BCA) and biliary cystadenocarcinoma (BCAC) are extremely rare neoplasms of the liver that share similar radiological characteristics (Wang et al., 2012). BCA accounts for $4.6 \%$ of intrahepatic cysts of bile duct origin, and most frequently occur in middle-aged women. BCAs are predominately benign tumors that have a low malignant potential (Billington et al., 2012).

There are three subsets of cystadenocarcinoma, which are based on pathology material submitted to institutional laboratories for primary diagnosis or consultation: 1) cystadenocarcinoma originating from a benign cystadenoma with ovarian-like stroma (occurs exclusively in women); 2) de novo cystadenocarcinoma occurring almost exclusively in men; and 3) cystadenocarcinoma in women without ovarian-like stroma.

The long list of possibilities in the differential diagnosis of BCA/BCAC includes simple cysts, parasitic cysts, degenerated metastatic tumors, mucin-producing metastatic tumors, congenital cystic dilation, cystic hemangioma, lymphangioma, hepatic foregut cyst, mesenchymal hamartoma, and teratoma (Hansman et al., 2001; Thomas et al., 2005; Del and Buonocore, 2008).

BCA is a premalignant lesion and cannot be reliably differentiated from BCAC by imaging. Therefore, both types of lesions should be excised (Ammori et al., 2002; Kubota et al., 2003; Koffron et al., 2004; Vogt et al., 2005). In this study, we reviewed our experience in the surgical management of patients who underwent operations for cystadenoma or cystadenocarcinoma at our institution in the last 10 years in an effort to formulate more effective management strategies.

\section{MATERIAL AND METHODS}

This study was approved by the Hepatobiliary Surgical Institute review board, Chinese PLA General Hospital, Beijing. We retrospectively reviewed clinical histories at our institution, and a dataset of patients examined for cystic liver lesions from January 1999 to December 2009 was compiled. Informed consent was obtained from all patients and approval was obtained from the designated review board of the institution.

The preoperative diagnosis of $\mathrm{BCA} / \mathrm{BCAC}$ was made based on radiological criteria [ultrasonography, computed tomography $(\mathrm{CT})$, or magnetic resonance imaging (MRI)]. Important radiological features were: 1) presence of a multilocular or unilocular mass with a well-defined capsule; 2) presence of one or more of the following structures exhibiting contrast enhancement: papillary projections, internal septations with nodular areas, wall thickness 
irregularities and mural nodules, and coarse calcifications along the wall (Lewin et al., 2006; Lim et al., 2007; Pojchamarnwiputh et al., 2008).

The postoperative diagnosis of BCA was made by pathological diagnosis (Koroglu et al., 2006; Zen et al., 2006): 1) macroscopically, their surface was usually flat, and may reach great proportions. The contents were mostly liquid, tending to become mucinous in texture, including biliary pigment, hemosiderine, and even purulent material if overinfected; and 2) under light microscopy, the inner surface was covered with a cuboidal-to-tall epithelium and some papillary and polypoid excrescences, basally oriented nuclei with a prominent nucleus and thick chromatin fibers, and pale acidophil cytoplasm with mucine-filled vacuoles.

Cyst fluid samples of 32 patients who were diagnosed with BCA/BCAC before the operation were obtained intra-operatively. We examined the level of carbohydrate associated antigen 19-9 (CA19-9) and carcinoembryonic antigen (CEA) in these samples. As a control group, we randomly extracted cyst fluid samples from 40 simple hepatic cyst patients, and examined their CA19-9 and CEA levels for statistical comparison.

Surgical intervention was performed if the patient was diagnosed with $\mathrm{BCA} / \mathrm{BCAC}$ based on CT or MRI. All specimens were sent for histopathological examination. The enucleation procedure was performed after non-malignancy diagnosis by frozen section.

All patients were followed by iconography for possible recurrences every six months in the first two postoperative years and then annually thereafter. These patients received a median of $20.45 \pm 8.2$ (range: 5-79) months of follow-up; one BCAC patient showed recurrence.

\section{RESULTS}

A total of 2297 cystic liver lesions patients from January 1999 to December 2009 were documented in our unit: 1813 patients had simple hepatic cysts, 410 patients had hepatic abscesses, 35 patients had parasitic cysts, and 39 patients had BCA/BCAC. All patients were reliably diagnosed based on postoperative pathological observations. The $39 \mathrm{BCA} / \mathrm{BCAC}$ patients $(\mathrm{N}=35 \mathrm{BCA}$ and $\mathrm{N}=4 \mathrm{BCAC})$ comprised 9 males and 30 females, with a median age of $53.74 \pm 14.50$ (range: 13-75) years. Therefore, BAC and BCAC accounted for $1.7 \%$ of all intrahepatic cystic liver lesions.

The most frequent symptom of all 39 BCA/BCAC patients was abdominal pain $(\mathrm{N}=$ 10, 25.7\%), abdominal mass $(\mathrm{N}=7,17.9 \%)$, abdominal distension $(\mathrm{N}=4,10.3 \%)$, obstructive jaundice $(\mathrm{N}=2,5.1 \%)$, and fever $(\mathrm{N}=3,7.7 \%)$. The remaining patients were asymptomatic $(\mathrm{N}=13,33.3 \%)$, and their cystic liver masses were discovered incidentally during radiological examinations (Table 1). One 54 year-old male patient with obstructive jaundice was particularly unique. His only typical symptom was obstructive jaundice, and he was preoperatively diagnosed with an intrahepatic bile duct stone. During the operation, however, we found a dense mucinous embolus obstructing the bile duct. His postoperative diagnosis was BAC (Figure 1).

In this study, the characteristic radiological features of BCA were based on a solitary, large, well-defined multilocular cystic lesion. Thick fibrous capsules and internal septations were often visible, and they helped to distinguish BCA lesions from simple cysts. Smooth and thin internal septa were also used as indicators of BCA (Figure 2). Calcification was uncommon, being observed in only three of the 39 cases, and was identified only in BCAC patients (Figure 3). 
Table 1. Clinic index of 39 cases diagnosed to biliary cystadenoma and biliary cystadenocarcinoma by postoperative pathological diagnosis.

\begin{tabular}{|c|c|c|}
\hline Clinic index & Number of cases & Percent $(\%)$ \\
\hline \multicolumn{3}{|l|}{ Gender } \\
\hline Male & 9 & 23.1 \\
\hline Female & 30 & 76.9 \\
\hline \multicolumn{3}{|l|}{ Symptom } \\
\hline Abdominal pain & 10 & 25.7 \\
\hline Abdominal mass & 7 & 17.9 \\
\hline Abdominal distension & 4 & 10.3 \\
\hline Obstructive jaundice & 2 & 5.1 \\
\hline Fever & 3 & 7.7 \\
\hline Absence of clinical symptoms & 13 & 33.3 \\
\hline \multicolumn{3}{|l|}{ Preoperative diagnosis } \\
\hline Cystadenoma & 29 & 74.4 \\
\hline Cystadenocarcinoma & 3 & 7.7 \\
\hline Hepatic abscess & 2 & 5.1 \\
\hline Intrahepatic bile duct stone & 1 & 2.6 \\
\hline Simple hepatic cysts & 4 & 10.3 \\
\hline \multicolumn{3}{|l|}{ Pathological diagnosis } \\
\hline Cystadenoma & 35 & 89.7 \\
\hline Cystadenocarcinoma & 4 & 10.3 \\
\hline \multicolumn{3}{|l|}{ Operative manner } \\
\hline Enucleation & 22 & 56.4 \\
\hline Left lateral lobe hepatectomy & 6 & 15.4 \\
\hline Left medial lobe hepatectomy & 3 & 7.7 \\
\hline Right hemi-hepatectomy & 4 & 10.3 \\
\hline Right anterior lobe hepatectomy & 2 & 5.1 \\
\hline Right posterior lobe hepatectomy & 2 & 5.1 \\
\hline \multicolumn{3}{|l|}{ Recurrence } \\
\hline Yes & 1 & 2.6 \\
\hline No & 38 & 97.4 \\
\hline
\end{tabular}
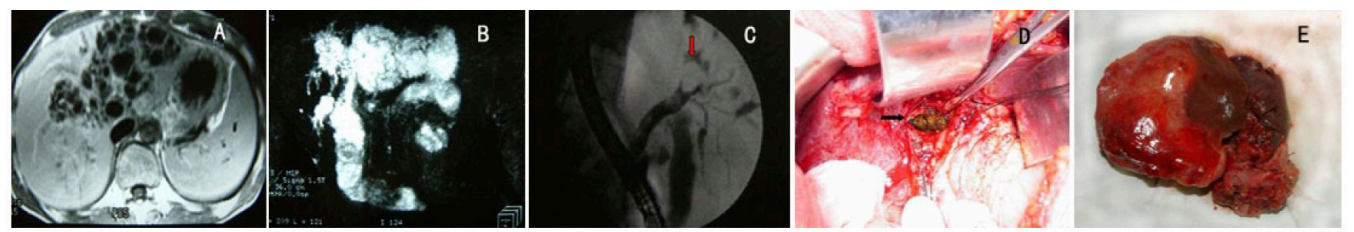

Figure 1. A. Magnetic resonance imaging showing different bile signals in left intrahepatic ducts in a multiloculated lesion with internal septation; B. magnetic resonance cholangiopancreatography showing the dilation of the left intrahepatic bile ducts; C. endoscopic retrograde cholangiopancreatography showing dilation of the left intrahepatic bile ducts and a tumor embolus in the left intrahepatic bile ducts; D. and E. macroscopy image revealing a multilocular cystic lesion containing serous fluid with tumor embolus protruding into the left hepatic duct.
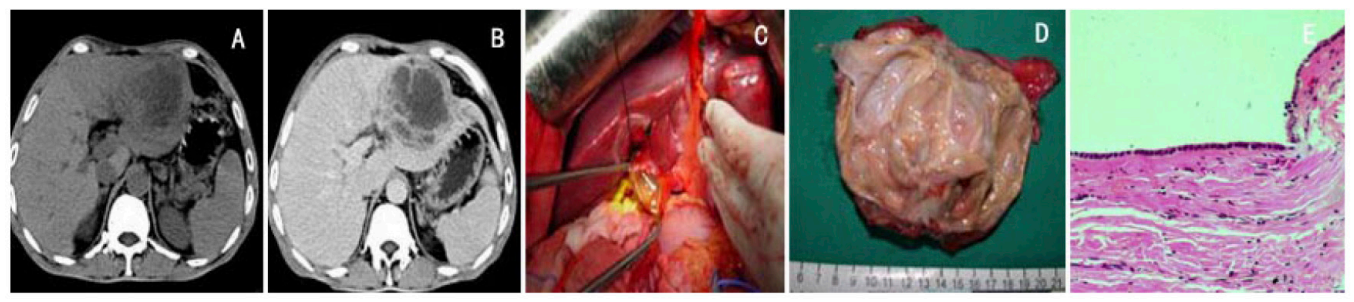

Figure 2. A. B. CT image showing a large cystic mass with a thick fibrous capsule with an enhanced mural nodule and internal septations in the left lateral lobe of the liver; C. viscous hyaline content in the cystic mass; D. gross photograph of the resected cystic mass showing a large opened cystic locule and several small cystic lesions. The cyst wall is smooth and glistening; E. photomicrograph of the epithelial lining on the cystic wall showing all columnar cells with bland-looking round nuclei (haematoxylin and eosin staining, 20X). 


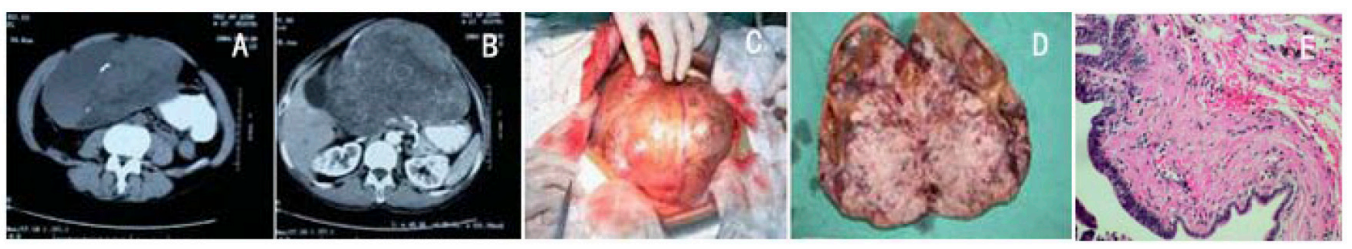

Figure 3. A. B. Calcifications along the wall of the neoplasm; C. D. multilocular cystic tumor protrudes to the surface of the left lateral lobe of the liver; E. pathological findings showing that the inner layer of the cyst is lined with a columnar epithelium exhibiting mild grade dysplasia along with a partial papillary epithelium.

CA19-9 and CEA levels were evaluated in cyst fluid samples from $32 \mathrm{BCA} / \mathrm{BCAC}$ patients and 40 simple hepatic cyst patients. The median CA19-9 value of the $32 \mathrm{BCA} / \mathrm{BCAC}$ samples was $364.79 \pm 47.26 \mathrm{U} / \mathrm{mL}$ (range: 0.33 to $8364.21 \mathrm{U} / \mathrm{mL}$ ). In 15 of the $32(46.9 \%)$ samples, CA19-9 levels were markedly increased relative to the normal range of serum (0.1$37 \mathrm{U} / \mathrm{mL}$ ). In the control simple hepatic cyst group, the CA19-9 median value was $21.37 \pm$ $14.35 \mathrm{U} / \mathrm{mL}$ (range: 0.60 to $185.92 \mathrm{U} / \mathrm{mL}$ ). Only four of the $40(10 \%)$ samples showed increased CA19-9 levels; both the median value and the percentage were statistically different $(\mathrm{P}<0.01)$. In the BCA/BCAC group, the CEA levels were increased in seven of $32(21.9 \%)$ samples (normal range of serum: $0-5 \mathrm{mg} / \mathrm{L}$ ), and the median value was $6.83 \pm 2.43 \mathrm{mg} / \mathrm{L}$ (range: 0.15 to $23.11 \mathrm{mg} / \mathrm{L}$ ). CEA levels were increased in five of the $40(12.5 \%)$ control simple hepatic cyst group samples; the median value was $4.21 \pm 2.91 \mathrm{mg} / \mathrm{L}$ (range: 0.27 to $36.21 \mathrm{mg} / \mathrm{L})$. The percentage was considered to be statistically different $(\mathrm{P}<0.01)$, but the median value was not $(\mathrm{P}>0.05)$ (Table 2$)$.

Table 2. Level of CA19-9 and CEA in cyst fluid of 32 BCA/BCAC cases and 40 simple hepatic cyst cases.

\begin{tabular}{|c|c|c|c|c|}
\hline & \multicolumn{2}{|c|}{$\mathrm{BCA} / \mathrm{BCAC}$ cases } & \multicolumn{2}{|c|}{ Simple hepatic cysts cases } \\
\hline & CA19-9 & CEA & CA19-9 & CEA \\
\hline Total of patients & 32 & 32 & 40 & 40 \\
\hline Median value (range) & $\begin{array}{c}364.79 \pm 47.26 \mathrm{U} / \mathrm{mL} \\
\text { (from } 0.33 \text { to } 8364.21 \mathrm{U} / \mathrm{mL} \text { ) }\end{array}$ & $\begin{array}{c}6.83 \pm 2.43 \mu \mathrm{g} / \mathrm{L} \\
\text { (from } 0.15 \text { to } 23.11 \mu \mathrm{g} / \mathrm{L} \text { ) }\end{array}$ & $\begin{array}{c}21.37 \pm 14.35 \mathrm{U} / \mathrm{mL} \\
\text { (from } 0.60 \text { to } 85.92 \mathrm{U} / \mathrm{mL} \text { ) }\end{array}$ & $\begin{array}{c}4.21 \pm 2.91 \mu \mathrm{g} / \mathrm{L} \\
\text { (from } 0.27 \text { to } 36.21 \mu \mathrm{g} / \mathrm{L} \text { ) }\end{array}$ \\
\hline $\begin{array}{l}\text { Number of patients whose } \\
\text { CA19-9 or CEA level increased } \\
\text { (normal range for serum) }\end{array}$ & $15(0.1-37 \mathrm{U} / \mathrm{mL})$ & $7(0-5 \mu \mathrm{g} / \mathrm{L})$ & $4(0.1-37 \mathrm{U} / \mathrm{mL})$ & $5(0-5 \mu \mathrm{g} / \mathrm{L})$ \\
\hline Percentage (\%) & 46.9 & 21.9 & 10 & 12.5 \\
\hline
\end{tabular}

$\overline{\mathrm{CEA}}=$ carcinoembryonic antigen; $\mathrm{BCA}=$ biliary cystadenoma; $\mathrm{BCAC}=$ biliary cystadenocarcinoma.

All the 39 patients with BCA or BCAC received surgical operation. Seven of the 40 patients were misdiagnosed before operation: four patients were operated with the preoperative diagnosis of simple hepatic cysts, two patients were diagnosed with hepatic abscesses, and one patient was diagnosed with an intrahepatic bile duct stone. Liver resection $(\mathrm{N}=17$, right hepatectomy in eight patients, left hepatectomy in nine patients) and enucleation ( $\mathrm{N}=$ 22) were performed. The final diagnosis was BCA in 35 patients and $\mathrm{BCAC}$ in four patients (Table 1).

All symptoms were resolved after surgery. There were no major complications or mortalities. All patients received a median of $20.45 \pm 8.2$ (range: $5-79$ ) months of follow up, and one patient showed recurrence. 


\section{DISCUSSION}

BCA and BCAC are rare biliary duct neoplasms. BCA is considered as a benign lesion with malignant potential, whereas BCAC is a malignant lesion (Mortelé and Ros, 2001). Although the incidence of BCA has been reported to be less than 5\% of all hepatic cystic lesions (Zen et al., 2006), this figure should be interpreted with caution due to the lack of criteria established for diagnosis and the large sample statistical data from a specialized institution. Therefore, the actual incidence of BAC/BCAC is probably much lower (Koffron et al., 2004). In the present study, BCA accounted for $1.7 \%$ of intrahepatic cystic liver lesions.

The clinical manifestations of these neoplasms are variable and nonspecific. Many of the patients were asymptomatic. The most typical symptoms were upper abdomen pain, abdominal mass, dyspepsia, anorexia, nausea and fever, jaundice caused by compression, protrusion, invasion of bile ducts, or secretion of dense mucinous material (Teoh et al., 2006).

In general, more than $80 \%$ of BCA and $63 \%$ of BCAC cases occur in women, and more than $80 \%$ are seen in patients older than 30 years of age. In the present study, $76.9 \%$ of BCA and $75 \%$ of BCAC occurred in women with a mean age of $53.74 \pm 14.50$ (range: 13-75) years.

The most widely used diagnosis methods are ultrasonography, CT, and MRI. The characteristic radiological feature of BCA and BCAC is usually a solitary, large, well-defined multilocular cystic lesion. A thick fibrous capsule and internal septations are often visible, and help to distinguish these lesions from a simple cyst. Finding smooth and thin internal septa is indicative of BCA. The presence of septations with nodularity or mural nodules is suggestive of BCAC. Although these mural nodules are seen in both BCA and BCAC, they occur more commonly in BCAC. Calcifications along the wall and internal septum are uncommon, and the presence of calcifications is suggestive of BCAC (Pojchamarnwiputh et al., 2008). In our study, although calcification was uncommon (7.7\%; three of the 39 cases), it was identified in BCAC cases only.

In spite of the improvements in imaging techniques and an increased awareness of these entities, the accurate preoperative diagnosis of these neoplasms remains difficult. The necessity and utility of obtaining cyst fluid aspiration for tumor marker (CEA or CA19-9) measurements has been a controversial issue until recently. The CA19-9 level of cystic fluid has been proposed "as a diagnostic help in liver cysts of unknown nature", and some centers have incorporated cyst fluid tumor marker (CA19-9 and CEA) measurements into their management algorithm (Koffron et al., 2004; Emre et al., 2011). However, definite diagnostic criteria for CA19-9 and CEA levels have not yet been established because the published data are largely limited to reports on increased marker levels in small numbers of BCA patients, without statistically robust comparison with levels in simple cysts. In our study, the CA199 median value and the percentage of patients with high CA19-9 levels in cystic fluid were markedly higher in the BCA/BCAC group than in the control simple hepatic cysts group ( $\mathrm{P}$ $<0.01$ ). The median value of CEA in cystic fluid of BCA/BCAC patients was only mildly increased relative to controls $(\mathrm{P}>0.05)$. Therefore, we regard the cystic fluid CA19-9 level as a diagnostic aid for $\mathrm{BCA} / \mathrm{BCAC}$ patients.

Treatment must be surgical whenever possible due to a potential malignant degeneration of these lesions. In our study, all patients received surgical resection, including liver resection or enucleation, and received a median $20.45 \pm 8.2$ months of follow-up; only one patient showed recurrence (recurrence rate $2.6 \%$ ). Surgical resection remains the treatment of choice 
for BCA/BCAC. Prognosis of BCA or BCAC is excellent if patients undergo a complete surgical resection in most cases. Follow-up with abdominal ultrasound or CT scans are suggested.

\section{CONCLUSIONS}

$\mathrm{BCA} / \mathrm{BCAC}$ are rare cystic neoplasms of the liver. Although their incidence has been reported to be less than $5 \%$ of all hepatic cystic lesions, the true incidence is probably much lower. In our study, the incidence of $\mathrm{BCA} / \mathrm{BCAC}$ was found to be $1.7 \%$ of all intrahepatic cystic liver lesions. The clinical manifestations of these neoplasms are variable and nonspecific. BCA is frequently misdiagnosed due to the lack of established criteria, and should therefore be suspected when a unilocular or multilocular cystic lesion with papillary infoldings is detected in the liver by iconographic examination. The presence of coarse calcifications is suggestive of BCAC. An increase in the CA19-9 level in cyst fluid is a helpful marker for distinguishing BCA/BCAC from common simple cysts. Surgical resection is the first choice for BCA/BCAC treatment. Prognoses were excellent after complete resection, and the recurrence rate was very low.

\section{Conflicts of interest}

The authors declare no conflict of interest.

\section{ACKNOWLEDGMENTS}

Research supported by the National Key Technology R\&D Program of China (\#2012BAI06B01).

\section{REFERENCES}

Ammori BJ, Jenkins BL, Lim PC, Prasad KR, et al. (2002). Surgical strategy for cystic diseases of the liver in a western hepatobiliary center. World J. Surg. 26: 462-469.

Billington PD, Prescott RJ and Lapsia S (2012). Diagnosis of a biliary cystadenoma demonstrating communication with the biliary system by MRI using a hepatocyte-specific contrast agent. Br. J. Radiol. 85: e35-e36.

Del PP and Buonocore M (2008). Cystic tumors of the liver: a practical approach. World J. Gastroenterol. 14: 3616-3620.

Emre A, Serin KR, Ozden I, Tekant Y, et al. (2011). Intrahepatic biliary cystic neoplasms: Surgical results of 9 patients and literature review. World J. Gastroenterol. 17: 361-365.

Hansman MF, Ryan JA, Jr., Holmes JH, Hogan S, et al. (2001). Management and long-term follow-up of hepatic cysts. Am. J. Surg. 181: 404-410.

Koffron A, Rao S, Ferrario M and Abecassis M (2004). Intrahepatic biliary cystadenoma: role of cyst fluid analysis and surgical management in the laparoscopic era. Surgery 136: 926-936.

Koroglu M, Akhan O, Akpinar E, Oto A, et al. (2006). Biliary cystadenoma and cystadenocarcinoma: two rare cystic liver lesions. JBR. -BTR. 89: 261-263.

Kubota E, Katsumi K, Iida M, Kishimoto A, et al. (2003). Biliary cystadenocarcinoma followed up as benign cystadenoma for 10 years. J. Gastroenterol. 38: 278-282.

Lewin M, Mourra N, Honigman I, Flejou JF, et al. (2006). Assessment of MRI and MRCP in diagnosis of biliary cystadenoma and cystadenocarcinoma. Eur. Radiol. 16: 407-413.

Lim JH, Jang KT, Rhim H, Kim YS, et al. (2007). Biliary cystic intraductal papillary mucinous tumor and cystadenoma/ cystadenocarcinoma: differentiation by CT. Abdom. Imaging. 32: 644-651.

Mortelé KJ and Ros PR (2001). Cystic focal liver lesions in the adult: differential CT and MR imaging features. Radiographics 21: 895-910.

Pojchamarnwiputh S, Na Chiangmai W, Chotirosniramit A and Lertprasertsuke N (2008). Computed tomography of 
biliary cystadenoma and biliary cystadenocarcinoma. Singapore Med. J. 49: 392-396.

Teoh AY, Ng SS, Lee KF and Lai PB (2006). Biliary cystadenoma and other complicated cystic lesions of the liver: diagnostic and therapeutic challenges. World J. Surg. 30: 1560-1566.

Thomas KT, Welch D, Trueblood A, Sulur P, et al. (2005). Effective treatment of biliary cystadenoma. Ann. Surg. 241: 769-773.

Vogt DP, Henderson JM and Chmielewski E (2005). Cystadenoma and cystadenocarcinoma of the liver: a single center experience. J. Am. Coll. Surg. 200: 727-733.

Wang C, Miao R, Liu H, Du X, et al. (2012). Intrahepatic biliary cystadenoma and cystadenocarcinoma: an experience of 30 cases. Dig. Liver Dis. 44: 426-431.

Zen Y, Fujii T, Itatsu K, Nakamura K, et al. (2006). Biliary cystic tumors with bile duct communication: a cystic variant of intraductal papillary neoplasm of the bile duct. Mod. Pathol. 19: 1243-1254. 